\title{
Avaliação e seleção precoce para crescimento de Pinus taeda ${ }^{(1)}$
}

\begin{abstract}
Estefano Paludzyszyn Filho(2), José Sebastião Cunha Fernandes ${ }^{(3)}$ e Marcos Deon Vilela de Resende ${ }^{(2)}$
Resumo - O objetivo deste trabalho foi avaliar a eficiência de diferentes métodos de seleção quanto aos caracteres ligados ao crescimento do fuste e o potencial da seleção precoce de genitores. Em 48 progênies de meios-irmãos de Pinus taeda L., foi medido o diâmetro do fuste a $30 \mathrm{~cm}$ e a $1,30 \mathrm{~m}$ do solo, aos 16 e 84 meses de idade, além da altura total. As progênies foram cultivadas em espaçamento tradicional e reduzido. Os componentes de variância, parâmetros e valores genéticos aditivos foram obtidos a partir de um modelo de análise individual. A seleção indireta foi de eficiência similar à direta quanto ao diâmetro (93\%) e menor quanto à altura (78\%). A seleção seqüencial foi 16\% (altura) e 28\% (diâmetro) mais eficiente que a seleção única. A eficiência da seleção combinada foi maior em relação a direta para diâmetro. Vinte genitores selecionados por valores genéticos aditivos quanto ao diâmetro, aos 16 e 84 meses, aumentaram a média em $7,6 \%$ e $8,1 \%$, respectivamente. A alta magnitude da correlação genética no que diz respeito ao diâmetro entre idades revelou que esse caráter, avaliado aos 16 meses de idade, prediz o crescimento aos 84 meses. Isso reduz custos de avaliação de progênies, antecipa o desbaste de genitores e a oferta de sementes melhoradas.
\end{abstract}

Termos para indexação: árvore florestal, correlação genética, ganho genético, melhoramento de plantas.

\section{Early evaluation and selection for growth in Pinus taeda}

\begin{abstract}
The objective of this work was to evaluate the efficiency of different methods of selection in growth traits and early selection. Individual total height and stem diameter at $30 \mathrm{~cm}$ above the ground in close-spaced early genetic evaluation at 16 months were assessed. In a 84 month sib cohort, as a comparison, height and diameter at breast height were evaluated. Phenotypic and genetic parameters for height and diameter were estimated for 48 open-pollinated families of loblolly pine (Pinus taeda L.) planted in Southern Brazil using an individual tree model. In this retrospective study, the indirect selection was of similar effectiveness as direct selection in stem diameter (93\%) and less effective in height (78\%). The two-stage successive culling process was $16 \%$ (height) and $28 \%$ (diameter) more efficient than the direct selection. The combined selection enhanced the accuracy of mature selection and increased expected genetic gain for diameter. Selecting 20 parents with the highest breeding values predicted by BLUP, at 16 and 84 months, attained a genetic gain of $7.6 \%$ and $8.1 \%$ in stem diameter. The strong age-age genetic correlation indicated that diameter was highly correlated to genetic level which implies that growth at 84 months could be improved by early selection of diameter at 16 months. This will provide quicker genetic gains by offering improved seeds earlier.
\end{abstract}

Index terms: forest trees, genetic correlation, genetic gain, plant breeding.

\section{Introdução}

Pinus taeda L. é a conífera que ocupa a maior área cultivada no sul-sudeste do Brasil. Isso se deve

\footnotetext{
(1) Aceito para publicação em 22 de julho de 2002.

(2) Embrapa-Centro Nacional de Pesquisa de Florestas, Caixa Postal 319, CEP 83411-000 Colombo, PR. E-mail: estefano@cnpf.embrapa.br, deon@cnpf.embrapa.br

(3) Setor de Ciências Agrárias/Fafeid, CEP 39100-000 Diamantina, MG. E-mail: cunha@citell.com.br
}

à produtividade e à qualidade da matéria-prima dessa espécie, muito usada na indústria de painéis de fibra de média densidade e na de celulose e papel. Nos plantios, são utilizadas mudas formadas a partir de sementes coletadas em matrizes fenotipicamente selecionadas. O longo intervalo entre as gerações de seleção, de aproximadamente dez anos (McKeand, 1988), é um grande entrave no melhoramento da espécie.

A seleção genética requer testes de longa duração, nos quais os caracteres de crescimento são ava- 
liados entre cinco e sete anos de idade (Balocchi et al., 1994). Esse período pode ser reduzido quando as árvores crescem e são avaliadas sob espaçamentos reduzidos $(0,5 \times 0,5 \mathrm{~m})$, em ambientes de solo o mais homogêneo possível (Bridgwater \& McKeand, 1997). Nessas condições, a altura do fuste, avaliada até os dois anos, permite predizer o desempenho das progênies entre oito e doze anos de idade em relação a esse caráter (Bridgwater \& McKeand, 1997). Nas mesmas condições, Williams \& Megraw (1994) constataram que a densidade básica aos dois anos pode ser utilizada na seleção de progênies. Testes de progênies com avaliações precoces podem ser retrospectivos de um teste de longa duração, o que possibilita correlacionar o desempenho das progênies quanto aos caracteres em diferentes idades.

A avaliação das progênies permite estimar os parâmetros fenotípicos e genéticos, predizer os valores genéticos em cruzamento, as relações entre os caracteres, bem como, avaliar a eficiência dos métodos de seleção e estimar os progressos genéticos pela seleção. Em P. taeda, a herdabilidade no sentido restrito quanto à altura é de magnitude moderada a alta (Balocchi et al., 1994). Em relação ao diâmetro, esse parâmetro é de magnitude moderada e menor do que o da altura (Foster, 1986; Gwaze et al., 2001). A correlação entre esses caracteres é positiva, e de magnitude moderada a alta (Bridgwater et al., 1983; Gwaze et al., 2001). Entre idades no mesmo caráter, no que diz respeito à altura e diâmetro, Gwaze et al. (2001) relatam que as correlações genéticas são de alta magnitude $(>0,80)$. Essas estimativas são mais precisas quando obtidas por modelos baseados no desempenho individual (Searle et al., 1992). Quando obtidos por meio de modelos bivariados, elas permitem estimar as relações entre os caracteres na mesma idade ou em idades diferentes, minorando efeitos do desbalanceamento dos dados.

Maior número de progênies pode ser avaliado sob espaçamentos reduzidos em ambientes de maior controle ambiental, como áreas agrícolas e casas de vegetação. Isso possibilita o aumento dos ganhos genéticos por geração de seleção (Wu et al., 1997), que pode ser visualizado pela eficiência da seleção estimada por diferentes métodos de seleção. Outro modo de conhecer o progresso pela seleção é por meio do uso dos valores genéticos dos genitores na seleção.
Para essas ações é necessário estimar parâmetros genéticos precisos, correlações entre caracteres em idade juvenil e adulta, e predizer os valores genéticos dos genitores.

O objetivo deste trabalho foi avaliar a eficiência de diferentes métodos de seleção quanto aos caracteres ligados ao crescimento do fuste e o potencial da seleção precoce de genitores.

\section{Material e Métodos}

\section{Avaliação em teste de longa duração}

Um teste com 48 progênies de meios-irmãos, obtidas de pomar clonal de matrizes selecionadas em plantios comerciais, no final da década de 70 (Fonseca et al., 1980), implantado pela Pisa Florestal S/A, em março de 1991, nos municípios de Sengés ( $24^{\circ} 3^{\prime}$ S) e Jaguariaíva ( $24^{\circ} 5^{\prime}$ S), no Estado do Paraná, foi utilizado para avaliar os caracteres diâmetro do fuste à altura do peito (DAP) e altura total das árvores (ALT), aos 84 meses de idade (idade adulta de seleção - IAS). Em experimentos com nove blocos completos casualizados, as progênies foram dispostas em parcelas lineares de seis plantas em espaçamento de $3 \times 2 \mathrm{~m}$. A bordadura do experimento foi dupla, formada com mudas oriundas dos excessos das progênies. Nenhuma adubação de manutenção ou reposição nas áreas experimentais foi realizada, até a idade de 84 meses.

\section{Avaliação em teste de curta duração}

Em novembro de 1997, as mesmas progênies do teste de longa duração foram plantadas na fazenda experimental da Embrapa em Ponta Grossa, PR, em espaçamento de 0,5x0,5 m, em Latossolo Vermelho-Amarelo, fase arenosa, dispostas em nove blocos completos casualizados, com parcelas lineares de seis plantas. Aos oito meses de idade, aplicaram-se a lanço $165 \mathrm{~kg} \mathrm{ha}^{-1}$ da fórmula 4-14-8. A área experimental foi mantida livre de ervas daninhas por capinas manuais, até os 16 meses de idade das plantas no campo, ocasião em que foi avaliado o diâmetro do fuste a $30 \mathrm{~cm}$ do solo (Diâm) e a altura total das plantas (Alt). Essa idade foi chamada de idade juvenil de seleção (IJS).

\section{Análises estatísticas}

A variação dos caracteres, altura total e diâmetro nos testes de curta e longa duração por local, foi analisada por modelo linear misto (modelo aditivo univariado), conforme Resende \& Fernandes (1999):

$\mathrm{y}=\mathrm{Xf}+\mathrm{Za}+\mathrm{Wp}+\mathrm{e}$

onde y é o vetor de dados do caráter; f é o vetor de efeitos fixos (média geral e blocos); a é o vetor de dados de efeitos 
genéticos aditivos (aleatórios) dos indivíduos avaliados; $\mathrm{p}$ é o vetor de efeito de parcelas (aleatório); e é o vetor de dados dos efeitos de erros aleatórios; X, Z e W são as matrizes de incidência para $f$, a e $p$, respectivamente.

$\mathrm{O}$ efeito da interação das progênies com locais $(\mathrm{Tg})$ dos experimentos na IAS foi verificado por um segundo modelo misto, conforme Resende (2000):

$\mathrm{y}=\mathrm{Xf}+\mathrm{Za}+\mathrm{Wp}+\mathrm{Tg}+\mathrm{e}$

onde $\mathrm{g}$ é o vetor de dados do efeito aleatório não-correlacionado da interação progênies com locais; fé o vetor de efeitos fixos com 18 blocos; T é a matriz de incidência para $g$.

Os componentes de variância e os parâmetros genéticos nos modelos 1 (aditivo univariado) e 2 (aditivo univariado ajustado) foram estimados por máxima verossimilhança restrita (REML), via algoritmo não-derivativo (DF), através do programa DFREML, versão 3.0 (Meyer, 1998), subprograma univariado (DFUNI).

A significância da diferença no ajuste dos diferentes modelos, que mede o efeito da interação das progênies com locais, foi avaliada pelo teste da razão de verossimilhança (LRT). Essa razão $(\lambda)$ foi comparada à função densidade de probabilidade (Tabela de qui-quadrado), com 1 grau de liberdade (Dobson, 1990). O LRT é dado por:

$\lambda=2\left(\log _{\mathrm{e}} \mathrm{L}_{2}-\log _{\mathrm{e}} \mathrm{L}_{1}\right)$

em que $\mathrm{L}_{1}$ e $\mathrm{L}_{2}$ são funções de verossimilhança estimadas pelos modelos 1 e 2 .

Considerando a não-significância da interação de progênies com locais dos caracteres avaliados na IAS, os dados foram agrupados, mantendo-se a sigla IAS.

A análise dos dados, na IJS e na IAS, de diâmetro e altura, seguiu o modelo 1, porém no plano bivariado, foi realizada conforme Resende et al. (1999). Esse modelo multivariado trata um mesmo caráter em diferentes locais ou estádios de desenvolvimento como diferentes caracteres (Resende, 2000), o que permite utilizar o subprograma multivariado (DXMUX) do programa DFREML.

As estimativas dos parâmetros, pelo modelo 1 (uni e bivariado), herdabilidade individual no sentido restrito $\left(\hat{\mathrm{h}}^{2}\right)$, coeficiente de variação genético aditivo $\left(\mathrm{CV}_{\mathrm{a}}\right)$, variância fenotípica $\left(\mathrm{s}^{2} \mathrm{~F}\right)$ e da correlação em decorrência do ambiente comum das parcelas $\left(\hat{c}^{2}\right)$ foram obtidas pelas expressões: $\hat{\mathrm{h}}^{2} \quad \hat{\sigma}_{\mathrm{a}}^{2} / \hat{\sigma}_{\mathrm{F}}^{2}$ : herdabilidade individual no sentido restrito no bloco;

$\hat{c}^{2} \quad \hat{\sigma}_{\mathrm{c}} / \hat{\sigma}_{\mathrm{F}}$ : correlação por causa do ambiente comum da parcela;

$\hat{\sigma}_{\mathrm{F}}^{2}=\hat{\sigma}_{\mathrm{a}}^{2}+\hat{\sigma}_{\mathrm{c}}^{2}+\hat{\sigma}_{\mathrm{e}}^{2}$ : variância fenotípica;
C Va $100\left(\hat{\sigma}_{a} / \bar{Y}\right)$ : coeficiente de variação genético aditivo,

onde $\overline{\mathrm{Y}}$ é a média geral; $\hat{\sigma}_{\mathrm{a}}^{2}$ é a variância genética aditiva; $\hat{\sigma}_{\mathrm{c}}^{2}$ é a variância entre parcelas; $\hat{\sigma}_{\mathrm{e}}^{2}$ é a variância residual (ambiental dentro de parcelas + não aditiva).

Utilizando o modelo bivariado, foram estimadas as correlações genéticas e fenotípicas entre as seguintes combinações de caracteres na IJS e IAS:

$\operatorname{Diâm}_{(\mathrm{IJS})}$ x DAP(IAS); $\operatorname{Alt}_{(\mathrm{IJS})}$ x $\mathrm{ALT}_{(\mathrm{IAS})}$; $\operatorname{Diâm}_{(\mathrm{IJS})}$ x $\mathrm{Alt}_{(\mathrm{IJS})}$; $\mathrm{DAP}_{(\mathrm{IAS})} \times \mathrm{ALT}_{(\mathrm{IAS})}$.

Entre caracteres, na mesma idade de seleção, a correlação genética aditiva $\left(r_{a}\right)$ e a fenotípica $\left(r_{f}\right)$ foram estimadas por:

$\hat{\mathrm{r}}_{\mathrm{a}(\mathrm{x}, \mathrm{y})}=\hat{\sigma}_{\mathrm{a}(\mathrm{x}, \mathrm{y})} /\left(\hat{\sigma}_{\mathrm{a}(\mathrm{x})} \hat{\sigma}_{\mathrm{a}(\mathrm{y})}\right)$;

$\hat{\mathrm{r}}_{\mathrm{F}(\mathrm{x}, \mathrm{y})}=\hat{\sigma}_{\mathrm{F}(\mathrm{x}, \mathrm{y})} /\left(\hat{\sigma}_{\mathrm{F}(\mathrm{x})} \hat{\sigma}_{\mathrm{F}(\mathrm{y})}\right)$,

em que $\hat{\sigma}_{\mathrm{a}(\mathrm{x}, \mathrm{y})}$ é a co-variância genética aditiva entre os caracteres x e y; $\hat{\sigma}_{\mathrm{F}(\mathrm{x}, \mathrm{y})}$ é a co-variância fenotípica entre os caracteres x e y; $\hat{\sigma}_{\mathrm{a}(\mathrm{x})}$ e $\hat{\sigma}_{\mathrm{a}(\mathrm{y})}$ são os desvios-padrões genéticos aditivos dos caracteres $\mathrm{x}$ e $\mathrm{y}$, respectivamente; $\hat{\sigma}_{\mathrm{F}(\mathrm{x})}$ e $\hat{\sigma}_{\mathrm{F}(\mathrm{y})}$ são os desvios-padrões fenotípicos dos caracteres $\mathrm{x}$ e $\mathrm{y}$, respectivamente.

Entre caracteres em diferentes indivíduos e idades, a correlação genética aditiva $\left(r_{a}\right)$ foi estimada a partir da correlação fenotípica:

$$
\begin{aligned}
& \hat{\mathrm{r}}_{\mathrm{F}(\mathrm{x}, \mathrm{y})}=\hat{\sigma}_{\mathrm{F}(\mathrm{x}, \mathrm{y})} /\left(\hat{\sigma}_{\mathrm{F}(\mathrm{x})} \hat{\sigma}_{\mathrm{F}(\mathrm{y})}\right) ; \\
& \hat{\mathrm{r}}_{\mathrm{a}(\mathrm{x}, \mathrm{y})}=\hat{\mathrm{r}}_{\mathrm{F}(\mathrm{x}, \mathrm{y})} /\left(\hat{\mathrm{h}}_{\mathrm{x}} \cdot \hat{\mathrm{h}}_{\mathrm{y}}\right),
\end{aligned}
$$

em que $\hat{\sigma}_{\mathrm{F}(\mathrm{x}, \mathrm{y})}$ é a co-variância fenotípica entre os caracteres x e y; $\hat{\sigma}_{\mathrm{F}(\mathrm{x})}$ e $\hat{\sigma}_{\mathrm{F}(\mathrm{y})}$ são os desvios-padrões fenotípicos dos caracteres $x$ e $y ; \hat{h}_{x}$ e $\hat{h}_{y}$ são a raiz quadrada da herdabilidade restrita dos caracteres x e y.

A eficiência da seleção genética das progênies, nos caracteres de crescimento nas diferentes idades, foi verificada por três métodos, detalhados a seguir.

\section{Método da seleção indireta}

A eficiência relativa (ER) da seleção precoce ou indireta, por ciclo de seleção ou de melhoramento na IJS, para melhorar o mesmo caráter na IAS, comparada à seleção com a mesma intensidade na IAS, foi computada conforme Falconer (1989):

$\mathrm{ER}=\left(\hat{\mathrm{h}}_{\text {IJS }} \hat{\mathrm{r}}_{\mathrm{a}(\mathrm{IJS}, \text { IAS })}\right) / \hat{\mathrm{h}}_{\text {IAS }}$,

em que $\hat{\mathrm{h}}_{\text {IJS }}$ e $\hat{\mathrm{h}}_{\text {IAS }}$ são a raiz quadrada das herdabilidades dos caracteres nas diferentes idades (estimativas do modelo linear misto, plano bivariado); 
$\mathrm{r}_{\mathrm{a}}$ é a correlação genética aditiva no mesmo caráter em diferentes idades de seleção.

Método de seleção seqüencial

A eficiência relativa (ER) da seleção seqüencial foi estimada com base nos parâmetros obtidos pelas avaliações na IJS e IAS. Na primeira etapa foi simulada a seleção de 32 dos 48 genitores. Na segunda, dos 32 genitores foram selecionados 20. Essas intensidades seguem práticas operacionais com a finalidade de manter a produção de sementes em volume considerável no pomar. A eficiência foi estimada conforme $\mathrm{Wu}$ (1998):

$$
\mathrm{ER}_{\text {(IIS-IAS/IAS) }}=\frac{\mathrm{i}_{\text {IJS }} \hat{\mathrm{h}}_{\text {IJS }}}{\mathrm{i}_{\text {IAS }} \hat{\mathrm{h}}_{\text {IAS }}} \mathrm{r}_{\mathrm{a}}+\frac{1-\hat{\mathrm{r}}_{\mathrm{a}}^{2} \hat{\mathrm{h}}_{\text {IJS }} \mathrm{k}}{\sqrt{1-\hat{\mathrm{r}}_{\mathrm{F}}^{2} \mathrm{k}}},
$$

em que $i_{\text {IJS }}$ e $i_{\text {IAS }}$ são as intensidades de seleção na IJS na IAS, respectivamente; $\mathrm{k}=\mathrm{i}_{\text {IJS }}\left(\mathrm{i}_{\text {IJS }}-\mathrm{a}\right) ; \mathrm{k}$ é o diferencial selecionado da população; a é o ponto de truncamento na curva normal padronizada, associado à intensidade de seleção na IJS.

\section{Método da seleção combinada}

A eficiência relativa da seleção combinada na IAS foi obtida pelos parâmetros estimados a partir das avaliações na IJS e IAS. Essa eficiência foi estimada conforme Resende \& Rosa-Perez (1999):

$\mathrm{ER}_{(\text {IJS,IAS/IAS) }}=\left[1+\frac{\left[\left(\frac{\hat{\mathrm{h}}_{\mathrm{IJS}}}{\overline{\mathrm{h}}_{\mathrm{IAS}}}\right) \hat{\mathrm{r}}_{\mathrm{a(IJS,IAS)}}-\hat{\mathrm{r}}_{\mathrm{F}(\mathrm{IJS}, \mathrm{IAS})}\right]^{2}}{1-\hat{\mathrm{r}}_{\mathrm{F}(\mathrm{IAS}, \mathrm{IJS})}^{2}}\right]^{1 / 2}$.

As herdabilidades para a seleção de progênies no âmbito de médias foram estimadas segundo Resende (1991).

O ganho genético pela seleção de genitores, por caracteres e idades, foi estimado a partir dos valores genéticos aditivos preditos pela "melhor predição linear não viciada" (BLUP), a partir do modelo misto 1, plano bivariado (modelo aditivo multivariado). A média dos maiores valores genéticos aditivos foi considerada como o incremento da média da próxima geração na idade de 84 meses.

\section{Resultados e Discussão}

A mortalidade nos testes de longa duração (IAS) em Sengés (2\%) e Jaguariaíva (8\%) foi, respectiva- mente, aleatória e localizada, e esta, provavelmente ocorreu por causa de danos provocados por animais de pequeno porte. No teste de curta duração (IJS), a perda de plantas foi de $8 \%$. Esses valores estão dentro dos esperados e, por serem baixos, não comprometem o número mínimo aceito para representar uma progênie de meios-irmãos.

Na IAS, as médias das progênies em diferentes locais para ALT e DAP não diferem entre si (Tabela 1). Na IJS, a altura média das progênies (Alt) é condizente com a observada por Bridgwater \& McKeand (1997) na mesma espécie, aos dois anos de idade. Com relação ao diâmetro do fuste aos $30 \mathrm{~cm}$ do solo (Diâm), em idades inferiores a dois anos, não há dados publicados.

Pelo modelo misto univariado, as estimativas dos coeficientes de herdabilidade, no sentido restrito, do DAP foram de magnitude similar entre os locais na IAS $(0,23$ e 0,19$)$, de magnitude assemelhada à do Diâm na IJS $(0,24)$ e de magnitude superior às obtidas por Bridgwater et al. (1983) e McKeand et al. (1997). No caráter altura, a herdabilidade diferiu entre locais e idades de seleção, indicando que a variação fenotípica do diâmetro não segue o mesmo padrão da altura. No entanto, essas estimativas foram superestimadas, por não ter sido possível separar a variância da interação progênie $\mathrm{x}$ ambiente, da variância da progênie.

$O$ coeficiente de variação genético aditivo $\left(\mathrm{CV}_{\mathrm{a}}\right)$ do DAP (IAS) foi de magnitude próxima da mediana

Tabela 1. Média geral, herdabilidade individual no sentido restrito no bloco e coeficiente de variação genético aditivo $\left(\mathrm{CV}_{\mathrm{a}}\right)$, dos caracteres altura total e diâmetro do fuste, avaliados em 48 progênies de meios-irmãos de Pinus taeda, aos 16 meses (IJS) e 84 meses (IAS) de idade.

\begin{tabular}{|c|c|c|c|}
\hline \multirow[t]{2}{*}{ Parâmetro } & \multicolumn{2}{|c|}{ IAS $^{(1)}$} & \multirow{2}{*}{$\begin{array}{c}\text { IJS }^{(2)} \\
\text { Ponta Grossa }\end{array}$} \\
\hline & Sengés & Jaguariaíva & \\
\hline & ------------D & (3) ------------- & Diâm $^{(4)}$ \\
\hline Média $(\mathrm{cm})$ & $15,5 \pm 3,14$ & $18,9 \pm 3,83$ & $2,1 \pm 0,49$ \\
\hline Herdabilidade & $0,23 \pm 0,05$ & $0,19 \pm 0,06$ & $0,24 \pm 0,06$ \\
\hline \multirow[t]{2}{*}{$\mathrm{CV}_{\mathrm{a}}(\%)$} & 9,7 & 8,9 & 11,4 \\
\hline & \multicolumn{2}{|c|}{-------------ALT ${ }^{(5)}$------------- } & $\mathrm{Alt}^{(5)}$ \\
\hline Média (m) & $9,73 \pm 1,27$ & $11,88 \pm 1,28$ & $2,0 \pm 0,3$ \\
\hline Herdabilidade & $0,32 \pm 0,06$ & $0,19 \pm 0,05$ & $0,14 \pm 0,04$ \\
\hline $\mathrm{CV}_{\mathrm{a}}(\%)$ & 7,3 & 5,6 & 5,5 \\
\hline
\end{tabular}

${ }^{(1)}$ Idade adulta de seleção. ${ }^{(2)}$ Idade juvenil de seleção. ${ }^{(3)}$ Diâmetro do fuste à altura do peito. ${ }^{(4)}$ Diâmetro do fuste a $30 \mathrm{~cm}$ do solo. ${ }^{(5)}$ Altura total do fuste. 
(8,6\%), de 58 estimativas ( $72 \%$ com Pinus spp.), apontada por Cornelius (1994). No Diâm (IJS), esse parâmetro foi de magnitude assemelhada. Isso indica que a contribuição do efeito de progênies ao efeito total da variação foi semelhante entre idades nesse caráter.

Esse mesmo coeficiente, no que tange a altura total, foi de magnitude menor na IJS em relação à IAS, o que evidencia que não houve melhoria do controle ambiental nesse caráter, nas progênies avaliadas em microambiente. A fertilização química, comumente não praticada nos plantios da espécie estudada, pode ter sido a causa da maior variação ambiental. Assim, as estimativas de parâmetros da população pela avaliação da altura das progênies na IJS devem ser restritas às condições deste estudo.

$\mathrm{O}$ ajuste do efeito da interação das progênies com os locais não foi significativo a $1 \%$ de probabilidade, verificado pela razão de verossimilhança, $\lambda=3,92$ $\mathrm{e} \lambda=1,07$, respectivamente, para ALT e DAP. Este fato permite o agrupamento de dados dos locais na IAS, esperando-se na seleção ganhos genéticos de maior precisão, pelo aumento da acurácia que mede o grau de confiança das estimativas. O aumento da acurácia é observado pela redução do desvio-padrão das estimativas, que ocorre quando se toma maior número de amostras por progênie (Hannrup et al., 1998).

Pelo modelo 2, plano bivariado, as estimativas de herdabilidade no sentido restrito para ALT e DAP foram de $0,30 \pm 0,04$ e $0,23 \pm 0,04$, respectivamente. Essa última tem magnitude moderada e similar à do modelo univariado (Tabela 1). Contudo, o aumento de amostras por progênies no que tange ao DAP, por agrupar os dados, reduziu o desvio-padrão da herdabilidade, conferindo maior precisão à estimativa. Essa redução também ocorreu quanto à ALT, a despeito das estimativas dos modelos uni e bivariado diferirem estatisticamente em Sengés. Tal fato ocorre, provavelmente, em razão da maior magnitude da interação das progênies com esse local, mesmo não sendo significativas. Na IJS, as estimativas de herdabilidade no que tange à Alt e Diâm pelo modelo bivariado foram $0,26 \mathrm{e} 0,21$, respectivamente.

As estimativas da herdabilidade, quanto ao diâmetro do fuste, não diferiram entre idades de seleção, revelando semelhante participação da variância genética aditiva na variância fenotípica do caráter nas idades. O mesmo não ocorreu com a altura. Isso indica que a seleção em relação ao diâmetro é mais promissora, como demonstrado pelo maior coeficiente de variação genética aditiva na IAS e IJS (Tabela 1), apesar da herdabilidade de menor magnitude quanto à altura. Além disso, a avaliação do diâmetro é mais precisa e de menor custo em relação à da altura, principalmente, na IAS.

A correlação genética aditiva dos mesmos caracteres em diferentes idades (age-age) foi de alta magnitude, de maior valor no diâmetro $(0,90)$ e menor na altura $(0,75)$, estando ambos de acordo com os resultados de Gwaze et al. (2001). Os desvios-padrões foram baixos, indicando que as estimativas das correlações genéticas são de alta precisão. Isso ocorre quando se utiliza elevado número de amostras (Klein et al., citado por Gwaze et al., 2001). Esses resultados indicam que a seleção precoce de progênies e genitores, em relação ao diâmetro e à altura, pode ser eficiente, apesar das progênies terem sido avaliadas em diferentes ambientes.

Na mesma idade de seleção, a correlação genética entre diâmetro e altura foi de magnitude positiva e alta $(>0,90)$ na IAS, o que está de acordo com os resultados de Bridgwater et al. (1983) e Gwaze et al. (2001). Também na IJS, a correlação genética entre esses caracteres foi de alta magnitude, em ambas as idades, o que indica que é esperada alta resposta correlacionada na seleção.

As correlações fenotípicas, nas combinações estudadas, foram de baixa magnitude, estando de acordo com Gwaze et al. (1997, 2001), indicando que esse parâmetro não deve ser utilizado para estimar os progressos pela seleção. Entretanto, foi observada alta correlação fenotípica entre DAP e $\operatorname{ALT}(0,78)$, o que evidencia que o efeito ambiental entre os caracteres nessa idade é maior (Tabela 2).

A eficiência da seleção indireta de progênies ou de genitores, pela avaliação na IJS, foi similar em relação ao diâmetro (93\%) e menos eficiente na altura (78\%), em relação à IAS (Tabela 3$)$. Isso sugere que, no caráter diâmetro, a discriminação precoce é efetiva, com pequeno sacrifício do progresso genético, porém, com ganho de tempo, fato importante pelo longo período da geração de $P$. taeda e por possibilitar a antecipação da oferta de sementes melhoradas pelo desbaste precoce de genitores, em pomar clonal. 
A eficiência da seleção seqüencial de progênies, em dois testes genéticos e idades, foi $16 \%$ e $28 \%$ maior para altura e diâmetro do fuste, respectivamente, do que a seleção única na IAS. Essa eficiência indica que é possível aumentar os ganhos genéticos em uma geração de seleção para ambos os caracteres.

A eficiência da seleção de progênies na IAS pelo método que combina os parâmetros da IJS e IAS foi $19 \%$ e $16 \%$ maior para altura e diâmetro, respectivamente (Tabela 3), do que a seleção única. Esse método aumenta a acurácia das estimativas de ganhos genéticos pelo maior número de informações das progênies.

A seleção de genitores do pomar clonal por valores genéticos aditivos preditos a partir da avaliação para o DAP aos 84 meses, com intensidade de $42 \%$, proporcionou a maior estimativa esperada de ganhos genéticos, elevando a média da população, em DAP, de 17,2 cm/árvore para 18,6 cm/árvore e de, 10,81 m/árvore para 11,22 m/árvore, em ALT

Tabela 2. Estimativas de correlações genética e fenotípica das combinações dos caracteres altura e diâmetro do fuste de 48 progênies de meios-irmãos de Pinus taeda, em diferentes idades de avaliação.

\begin{tabular}{lcc}
\hline Caracteres & \multicolumn{2}{c}{ Estimativas da correlação } \\
\cline { 2 - 3 } & \multicolumn{3}{c}{ Genética aditiva } & Fenotípica \\
\hline \multirow{2}{*}{ Diâm $^{(1)} \times$ DAP } & Diferentes idades de seleção \\
Alt $^{(3)} \times \mathrm{ALT}^{(4)}$ & $0,90 \pm 0,03$ & 0,22 \\
& $0,75 \pm 0,04$ & 0,19 \\
\hline \multirow{3}{*}{ Alt x Diâm } & \multicolumn{1}{c}{ Na mesma idade de seleção } \\
ALT x DAP & $0,90 \pm 0,04$ & 0,23 \\
\hline
\end{tabular}

(1)Diâmetro do fuste a $30 \mathrm{~cm}$ do solo avaliado no teste da idade juvenil de seleção (IJS, 16 meses). ${ }^{(2)}$ Diâmetro do fuste na altura do peito avaliado nos testes da idade adulta de seleção (IAS, 84 meses). ${ }^{(3)}$ Altura total do fuste na IJS. ${ }^{(4)}$ Altura total do fuste na IAS.

Tabela 3. Eficiência relativa de diferentes métodos de seleção de progênies de Pinus taeda, por geração de melhoramento para diâmetro na altura do peito e altura total do fuste.

\begin{tabular}{llcc}
\hline Idade $^{(1)}$ & $\begin{array}{c}\text { Método de } \\
\text { seleção }\end{array}$ & \multicolumn{2}{c}{$\begin{array}{c}\text { Eficiência relativa (\%) em relação } \\
\text { à seleção única na IAS (100\%) }\end{array}$} \\
\cline { 3 - 4 } & & Altura & Diâmetro \\
\hline IJS & Indireto & 78 & 93 \\
IJS + IAS & Seqüencial $^{(2)}$ & 116 & 128 \\
IAS & Combinada $^{2}$ & 119 & 116 \\
\hline
\end{tabular}

(1) IJS: idade juvenil de seleção (16 meses); IAS: idade adulta de seleção (84 meses). ${ }^{(2)}$ Intensidade de seleção de $66 \%$ na IJS e na IAS.
(Tabela 4). Níveis semelhantes de aumento do crescimento por árvore podem ser obtidos por meio de seleção em altura. No entanto, o caráter DAP apresenta maiores facilidades operacionais de avaliação.

A seleção de 20 genitores em 48 do pomar clonal, por valores genéticos aditivos (VG's) preditos a partir da avaliação do diâmetro das progênies a $30 \mathrm{~cm}$ do solo aos 16 meses, proporcionou, em média, ganhos esperados muito próximos dos esperados por VG's preditos a partir da avaliação do DAP. Este fato era esperado porque os genitores selecionados por VG's nas diferentes idades foram os mesmos, com exceção de um.

A mesma intensidade de seleção, com relação à altura do fuste pelos VG's das progênies aos 16 meses, resultou em um ganho genético esperado superior ao dos VG's preditos pela avaliação do DAP. Isso é devido ao fato dos genitores selecionados entre as diferentes idades não serem os mesmos, provavelmente, pela avaliação menos precisa da altura. Esses resultados revelam que a seleção precoce é eficiente e os ganhos esperados são semelhantes aos da seleção direta nos caracteres de crescimento. Além disso, é possível dispor de sementes melhoradas em menor prazo. Assim, em apenas uma geração de seleção, ganhos genéticos maiores são esperados em P. taeda com menor investimento em testes tradicionais. Para isso, um maior número de progênies deve ser precocemente avaliado.

Tabela 4. Ganho genético aos 84 meses de idade em altura e diâmetro do fuste pela seleção direta na idade adulta de seleção (IAS) e indireta na idade juvenil de seleção (IJS), por valores genéticos aditivos (VG's) de 20 genitores da avaliação das progênies aos 16 e 84 meses de idade ${ }^{(1)}$.

\begin{tabular}{lccccc}
\hline $\begin{array}{l}\text { Caráter } \\
\text { selecionado }\end{array}$ & \multicolumn{2}{c}{$\begin{array}{c}\text { Média dos 20 } \\
\text { maiores VG's no } \\
\text { caráter resposta }\end{array}$} & & \multicolumn{2}{c}{$\begin{array}{c}\text { Nova média do caráter e } \\
\text { ganhos }(\%)\end{array}$} \\
\cline { 2 - 3 } \cline { 5 - 6 } & $\begin{array}{c}\text { DAP } \\
(\mathrm{cm})\end{array}$ & $\begin{array}{c}\text { ALT } \\
(\mathrm{m})\end{array}$ & & $\begin{array}{c}\text { DAP } \\
(\mathrm{cm})\end{array}$ & $\begin{array}{c}\text { ALT } \\
(\mathrm{m})\end{array}$ \\
\hline \multirow{3}{*}{ DAP } & 1,4 & 0,41 & & $18,6(8,1)$ & $11,22(3,8)$ \\
ALT & 0,4 & 0,75 & & $17,6(2,6)$ & $11,56(7)$ \\
\hline \multicolumn{5}{c}{ Idade adulta de seleção } \\
Diâm & 1,3 & 0,39 & & $18,5(7,6)$ & $11,19(3,6)$ \\
Alt & 1,1 & 0,53 & $18,3(6,5)$ & $11,33(4,9)$ \\
\hline
\end{tabular}

(1)DAP: diâmetro do fuste na altura do peito (1,30 m do solo); ALT: altura total do fuste; Diâm: diâmetro do fuste a $30 \mathrm{~cm}$ do solo; Alt: altura total do fuste aos 16 meses de idade. 


\section{Conclusões}

1. Nos testes de progênies, na idade de 84 meses, ocorrem efeitos de locais sobre a média geral das progênies nos caracteres DAP e ALT.

2. Os caracteres diâmetro e altura do fuste apresentam herdabilidade de magnitude moderada nas avaliações aos 16 e 84 meses, revelando boas perspectivas de resposta à seleção, melhor no caráter diâmetro.

3. Maior ganho genético por geração de seleção é obtido pelo emprego do método seqüencial, principalmente no diâmetro do fuste, em virtude da maior magnitude dos coeficientes de correlação genética aditiva e dos menores desvios-padrão para esse caráter em diferentes idades.

4. Os ganhos pela seleção de genitores por valores genéticos aditivos, preditos para diâmetro do fuste aos 16 e 84 meses de idade, foram similares e revelam o potencial da seleção precoce.

5. O maior progresso genético pela seleção na idade de 84 meses para crescimento não supera o benefício da oferta antecipada de sementes melhoradas.

\section{Referências}

BALOCCHI, C. E.; BRIDGWATER, F. E.; BRYANT, R. Selection efficiency for a non-selected population of loblolly pine. Forest Science, Bethesda, v. 40, n. 3, p. 452-473, 1994.

BRIDGWATER, F. E.; McKEAND, S. E. Early family evaluation for growth of loblolly pine. Forest Genetics, Zvolen, v. 4, n. 1, p. 51-58, 1997.

BRIDGWATER, F. E.; TALBERT, J. T.; JAHROMI, S. Index selection for increased dry weight in a young loblolly pine population. Silvae Genetica, Frankfurt, v. 32, n. 5/6, p. 157-161, 1983.

CORNELIUS, J. Heritabilities and additive genetic coefficients of variation in forest trees. Canadian Journal of Forest Research, Ottawa, v. 24, p. 372-379, 1994.

DOBSON, A. J. An introduction to generalized linear models. Melbourne: Chapman \& Hall, 1990. 174 p.

FALCONER, D. S. Introduction to quantitative genetics. 3. ed. Harlow: Longman, 1989. 438 p.

FONSECA, S. M.; KAGEYAMA, P. Y.; FERREIRA, M.; JACOB, W. S. Programa conjunto de melhoramento ge- nético e produção de sementes de Pinus taeda na Região Sul do Brasil. Boletim Informativo IPEF, Piracicaba, v. 6, n. 48 , p. 61-73, 1980.

FOSTER, G. S. Trends in genetic parameters with stand development and their influence on early selection for volume growth in loblolly pine. Forest Science, Bethesda, v. 32, n. 4, p. 944-959, 1986.

GWAZE, D. P.; BRIDGWATER, F. E.; BYRAM, T. D.; LOWE, W. J. Genetic parameter estimates for growth and wood density in loblolly pine (Pinus taeda L.). Forest Genetics, Zvolen, v. 8, n. 1, p. 47-55, 2001.

GWAZE, D. P.; WOOLLIAMS, J. A.; KANOWSKI, P. J. Optimum selection age for height in Pinus taeda L. in Zimbabwe. Silvae Genetica, Frankfurt, v. 46, n. 6, p. 358-365, 1997.

HANNRUP, B.; WILHELMSSON, L.; DANELL, O. Time trends for genetic parameters of wood density and growth traits in Pinus sylvestris L. Silvae Genetica, Frankfurt, v. 47, n. 4, p. 214-219, 1998.

McKEAND, S. E. Optimum age for family selection for growth in genetic tests of loblolly pine. Forest Science, Bethesda, v. 34, n. 2, p. 400-411, 1988.

McKEAND, S. E.; ERICKSSON, G.; ROBERDS, J. H. Genotype by environment interaction for index traits that combine growth and wood density in loblolly pine. Theoretical and Applied Genetics, Berlin, v. 94, p. 1015-1022, 1997.

MEYER, K. DFREML: version 3.0 b user notes. Edinburgh: Institute of Animal Genetics of Edinburgh/Armidale:University of New England, Animal Genetics and Breeding Unit, 1998. 31 p.

RESENDE, M. D. V. de. Análise estatística de modelos mistos via REML/BLUP na experimentação em melhoramento de plantas perenes. Colombo: Embrapa-CNPF, 2000. 101 p. (Documentos, 47).

RESENDE, M. D. V. de. Correções nas expressões do progresso genético com seleção em função da amostragem finita dentro de famílias e populações e implicações no melhoramento florestal. Boletim de Pesquisa Florestal, Colombo, v. 22/23, p. 61-77, 1991.

RESENDE, M. D. V. de; FERNANDES, J. S. C. Procedimento BLUP individual para delineamentos experimentais aplicados ao melhoramento florestal. Revista de Matemática e Estatística, São Paulo, v. 17, p. 89-107, 1999.

RESENDE, M. D. V. de; FERNANDES, J. S. C.; SIMEÃO, R. M. BLUP individual multivariado em pre- 
sença de interação genótipo $\mathrm{x}$ ambiente para delineamentos experimentais repetidos em vários ambientes. Revista de Matemática e Estatística, São Paulo, v. 17, p. 209-228, 1999.

RESENDE, M. D. V. de; ROSA-PEREZ, J. R. H. Genética quantitativa e estatística no melhoramento animal. Curitiba: UFPR, 1999. 494 p.

SEARLE, S. R.; CASELlA, G.; McCULLOCH, C. E. Variance components. New York: J. Wiley, 1992. 528 p.

WILLIAMS, C. G.; MEGRAW, R. A. Juvenile-mature relationship for wood density in Pinus taeda. Canadian
Journal of Forest Research, Ottawa, v. 24, p. 714-722, 1994.

WU, H. X. Study of early selection in tree breeding 1. Advantage of early selection through increase of selection intensity and reduction of field test size. Silvae Genetica, Frankfurt, v. 47, n. 2/3, p. 146-155, 1998.

WU, H. X.; YEH, F. C.; DHIR, N. K.; PHARIS, R. P.; DANCIK, B. P. Genotype by environment interaction and genetic correlation of greenhouse and field performance in Pinus contorta ssp. latifolia. Silvae Genetica, Frankfurt, v. 46, p. 170-175, 1997. 